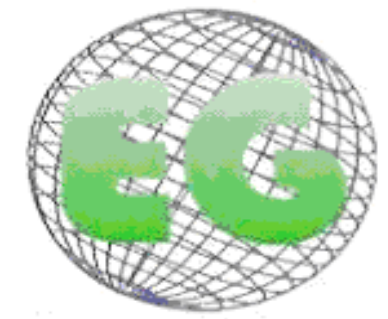

ISSN 1695-6141

N24

\title{
Producción científica enfermera del Hospital Infanta Elena de Huelva durante los años 1985-2009
}

Nursing scientific production in the "Infanta Elena" Hospital (Huelva) during the years 1985-2009

\section{*Lozano León, T. ${ }^{* *}$ Minchón Hernando, A., "***Romero Indiano, EM.}

\author{
*Doctorando en Enfermería. Distrito Sanitario Condado-Campiña, ** Diplomado en Enfermería. Experto en \\ Gestión Sanitaria. Hospital Juan Ramón Jiménez. *** Diplomada en Enfermería. Distrito Sanitario Condado-
} Campiña. Huelva.

(Premio a la Mejor Comunicación Oral en las XII Jornadas de Enfermería de la ADEHIE (Asociación de Enfermería del Hospital Infanta Elena de Huelva celebradas el 25 y 26 de noviembre de 2010)

Palabras clave: Bibliometría; Enfermería; Indicadores de Producción Científica; Investigación en Enfermería; Publicaciones Científicas.

Keywords: Bibliometrics; Nursing; Nursing Research; Scientific Publication Indicators; Scientific Publication.

\section{RESUMEN}

El presente trabajo tiene como finalidad describir la producción científica del personal de Enfermería del Hospital Infanta Elena de Huelva durante los años 1985 al 2009. Para ello se plantea una estrategia de búsqueda en la base de datos Cuiden Plus y se extraen todas las referencias bibliográficas que cumplen las condiciones descritas. Se recuperan un total de 68 documentos. El $35 \%$ de los artículos se publican en revistas de ámbito local. El $80 \%$ se publica en revistas sin Impacto. El $65 \%$ de los documentos son citables (originales 0 revisión). Un total de 98 autores firman todos los artículos, de los que el 67 son productores ocasionales. La temática más habitual se centra en la Enfermería y en los cuidados de Enfermería. Hay una escasa colaboración tanto institucional como a nivel de autoría. Se constata un crecimiento exponencial de las publicaciones.

\section{ABSTRACT}

The aim of this paper is to describe the scientific production of nursing staff of the "Infanta Elena" Hospital (Huelva) for the years 1985 to 2009. We proposed a search strategy in the Cuiden Plus database and extracted all references to the conditions described. 68 documents were recovered. $35 \%$ of the articles were published in local journals. $80 \%$ were published in journals without impact. $65 \%$ of the documents are citable (original or review). A total of 98 authors have signed all the papers, of which 67 are occasional producers. The most common theme focuses on Nursing and Nursing Care. There is a lack of cooperation both at the institutionally and in terms of authorship. There has been an exponential growth of publications. 


\section{INTRODUCCIÓN}

El conocimiento científico, como acervo de la humanidad, aumenta y se enriquece cuando quienes lo generan publican el resultado de sus investigaciones. El medio más aceptado para ello en el mundo académico es el artículo científico. Las revistas científicas constituyen el instrumento imprescindible para impulsar la difusión social del conocimiento científico y tecnológico. ${ }^{1}$

Sin embargo la creación de este producto final es parte de un fenómeno más amplio denominado ciclo de la producción científica, según el cual, el artículo científico publicado permite a la comunidad científica conocer y contrastar los hallazgos, ${ }^{2}$ incorporarla al conocimiento disciplinar y, en último lugar, utilizarla en la construcción teórica o práctica de la Ciencia mediante la cita y uso del documento en futuros productos. ${ }^{3}$ La cita del documento por otros autores es el punto final del ciclo de la producción científica, y da lugar al inicio de un nuevo ciclo para el documento que se citado. ${ }^{4}$

La producción científica no puede avanzar sino apoyándose en los trabajos precedentes, siendo los trabajos actuales la base de futuras investigaciones y publicaciones.

El análisis de la publicación de artículos científicos es un eslabón fundamental dentro del proceso de investigación, se ha convertido en la unidad estándar que permite calificar la calidad del proceso generador de conocimiento y su impacto en el entorno científico. ${ }^{5}$

La bibliometría es la ciencia que permite el análisis cuantitativo de la producción científica a través de la literatura, estudiando la naturaleza y el curso de una disciplina científica ${ }^{6-9}$. Los investigadores López Piñero y Terrada fueron los primeros que introdujeron los estudios bibliométricos en España hace ya tres décadas y desde entonces se han realizado interesantes estudios sobre la producción científica española en biomedicina y salud ${ }^{10,11}$.

La producción científica se estudia a través de los documentos de las revistas, localizados manualmente o bien recuperados a partir de bases de datos; mediante el análisis de los diversos indicadores de producción, circulación, dispersión, consumo de la información y repercusión.

La bibliometría es un instrumento esencial para evaluar el producto de la ciencia y en el caso de disciplinas incipientes en materia de investigación, como es la enfermería, es una herramienta esencial para determinar su avance y estado de consolidación.

Son varios los estudios de este tipo los que nos encontramos en la literatura científica relacionado con la Enfermería; sobre todo los que hacen referencia al análisis de producción de revistas científicas ${ }^{12-17}$ o de diversas disciplinas, pero hasta ahora no ha podido recuperar ningún documento que centre su análisis en una institución concreta.

En cuanto a las bases de datos especializadas en Enfermería, Cuiden, perteneciente a la Fundación Index de Granada es la más importante ${ }^{18}$. Contiene 464 publicaciones, 102 de las 116 revistas de Enfermería españolas están indizadas en Cuiden, todas las revistas de Enfermería españolas con Factor de Impacto están incluidas en esta base de datos.

El presente trabajo tiene como finalidad describir la producción científica del personal de Enfermería del Hospital Infanta Elena de Huelva durante los años 1985 al 2009 a partir de 
las referencias bibliográficas recuperadas de la base de datos Cuiden Plus perteneciente a la Fundación Index.

\section{MATERIAL Y MÉTODO}

Diseño: Estudio Bibliométrico descriptivo transversal.

Fuente de datos: Se realiza búsqueda bibliográfica el 01/11/2010 en la Base de Datos CUIDEN.

Estrategia de búsqueda: Se ha realizado la búsqueda en la base de datos con la siguiente ecuación de búsqueda:

- Keywords: HOSPITAL INFANTA ELENA.

- Campo: Autores/Institución/Función.

- Workform: ARTICULO OR ACTAS DE CONGRESO OR CAPITULO DE LIBRO OR LIBRO OR LITERATURA GRIS OR MATERIAL AUDIOVISUAL.

- Año de publicación desde 1985 hasta 2009.

Población de estudio: Todos los artículos incluidos en la base de datos Cuiden Plus a partir de la ecuación de búsqueda anterior.

Variables e indicadores:

- Número de documentos. Documentos citables. Thomson Reuters (Antes ISI) considera los documentos susceptibles de ser citados los artículos originales, notas y revisiones.

- Año de publicación.

- Revistas fuente. Ámbito de la revista: Local, nacional o internacional.

- Repercusión (Impacto). Para la distribución de la publicación por Impacto se utiliza el indicador Repercusión Inmediata Cuiden (RIC) que es el número de citas que recibe una revista dividido entre el número de artículos publicados. A su vez distribuidos en cuartiles.

- Tipo de artículo: según la distribución tipológica de la Fundación Index.

- Autoría: número de autores. Autores por trabajo y colaboración. Productividad.

- Temática: Áreas más relevantes según la distribución de las palabras clave.

- Idioma.

Análisis: Se diseña matriz de datos ad hoc y se exporta a SPSS. Se realiza un análisis descriptivo de los datos a través de tablas de frecuencias y gráficos.

Criterios Inclusión:

- Artículos indizados en la base de datos Cuiden en los años 1985-2009.

- Autoría: Institución Hospital Infanta Elena de Huelva.

- Al menos uno de los autores Enfermero/a.

Criterios Exclusión:

- Comunicaciones a congresos tanto orales como pósteres.

- Ponencias. 
- Noticias.

- Reseñas bibliográficas.

- Capítulos de libros o libros completos.

\section{RESULTADOS}

Se han recuperado un total de 67 Artículos y 3 Actas de Congresos con la ecuación de búsqueda anteriormente descrita. Una vez eliminados los redundantes y los no pertinentes se obtienen 68 documentos publicados por autores firmantes de la institución Hospital Infanta Elena de Huelva en el período 1985-2009. El 56\% de los documentos son citables.

El 35\% de la producción científica se publica entre los años 2006 y 2009 . El 53\% de documentos tienen más de 10 años de antigüedad. Esto supone una producción de 2.72 documentos al año, a pesar que su distribución anual es muy irregular. (Gráfico 1)

Gráfico 1: Producción anual HIE.

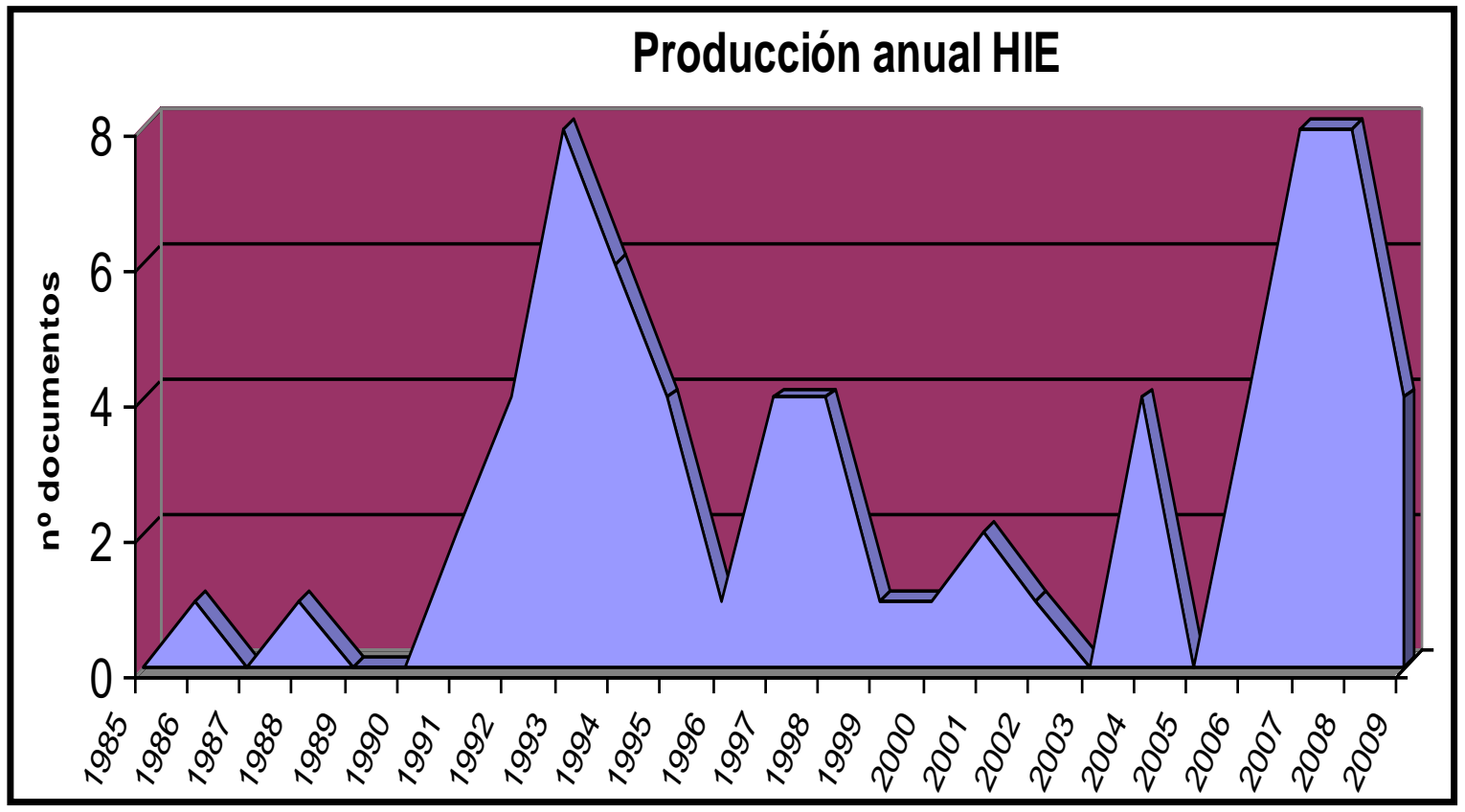

El 35\% de los artículos se publican en revistas de ámbito local (Documentos de Enfermería o Sesiones de Salud). No se publica ningún artículo en revistas de ámbito internacional. Las revistas Rev AE Enferm Urolog y Rol de Enfermería publican 6 documentos cada una en el período de estudio. (Gráfico 2) 
Gráfico 2: Distribución de las publicaciones por revistas fuente.

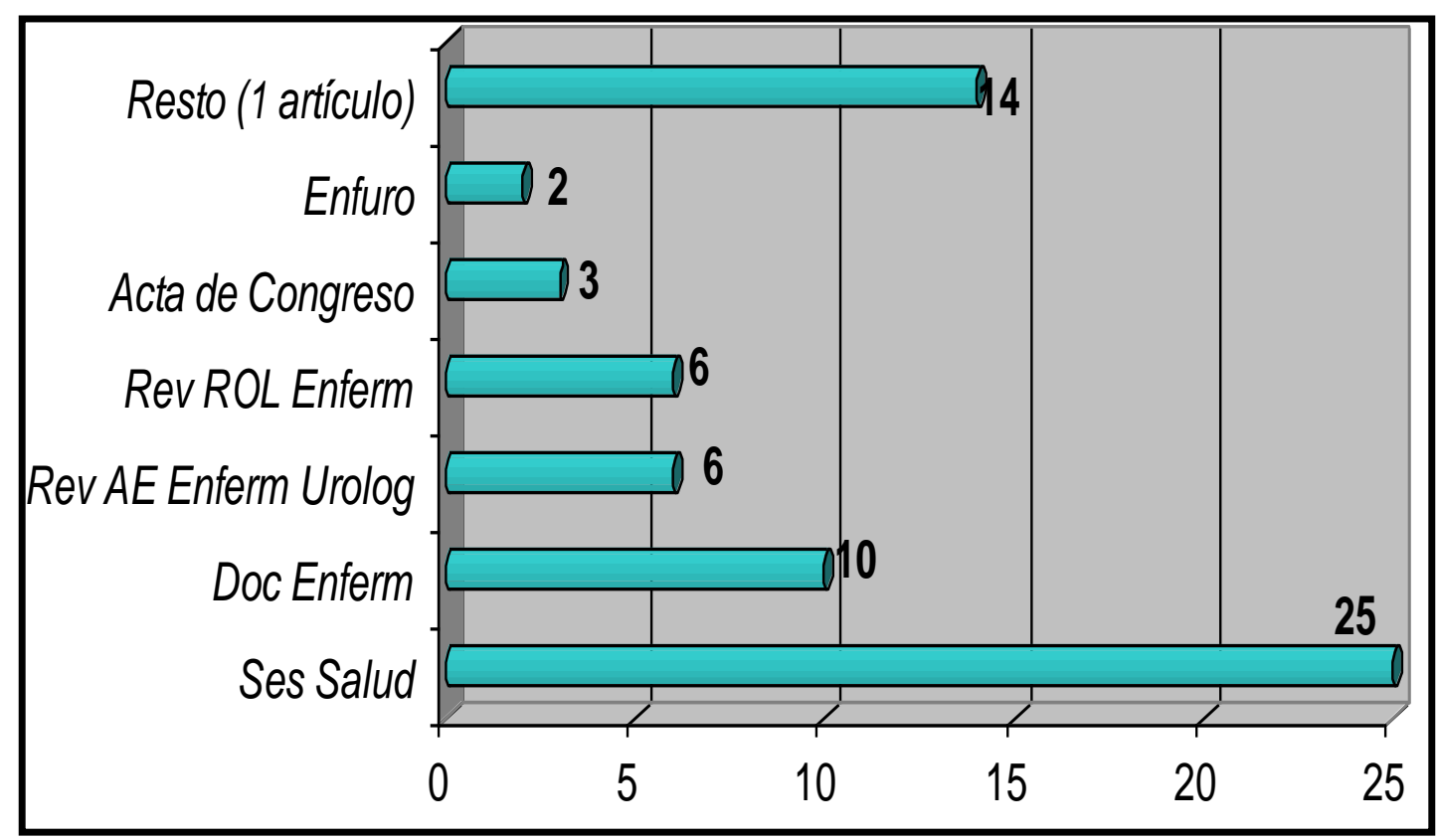

Se publica un artículo en revista situada en el primer cuartil de Repercusión Cuiden (Impacto) y 7 artículos en el segundo cuartil. El $80 \%$ de los documentos se publican en revistas sin Impacto. (Tabla 1)

Los tipos de documentos más publicados son originales, de revisión y protocolos y pautas de actuación que suman un $65 \%$ del total. (Tabla 2)

Tabla 1: Distribución de los artículos por el Impacto de la revista.

\begin{tabular}{|c|c|c|}
\hline Cuartil & Título de la revista & Artículos \\
\hline 1 & Index de Enfermería & 1 \\
\hline 2 & Evidentia & 1 \\
\hline \multirow{3}{*}{3} & Revista Rol de Enfermería & 6 \\
\hline & Enferm Comunitaria & 1 \\
\hline & Archivos de la Memoria & 1 \\
\hline \multirow[t]{3}{*}{4} & Tesela & 1 \\
\hline & Enfermería Global & 1 \\
\hline & Sin Impacto & 56 \\
\hline
\end{tabular}


Tabla 2: Distribución de artículos según su tipología documental.

\begin{tabular}{|lc}
\hline Tipología documental & Frecuencia \\
Artículo Original & 21 \\
Artículo de revisión & 17 \\
Protocolo y pautas de actuación & 12 \\
Comunicación & 6 \\
Artículo Breve & 4 \\
Experiencias y traba & 3 \\
Caso clínico & 2 \\
Artículo Histórico & 1 \\
Guías y programas & 1 \\
Relato biográfico & 1 \\
Total & 68
\end{tabular}

Se contabilizan 98 firmantes que computan un índice de colaboración de 2,4. La mayoría de los autores $67 \%$ son productores ocasionales que sólo firman 1 documento (Gráfico 3).

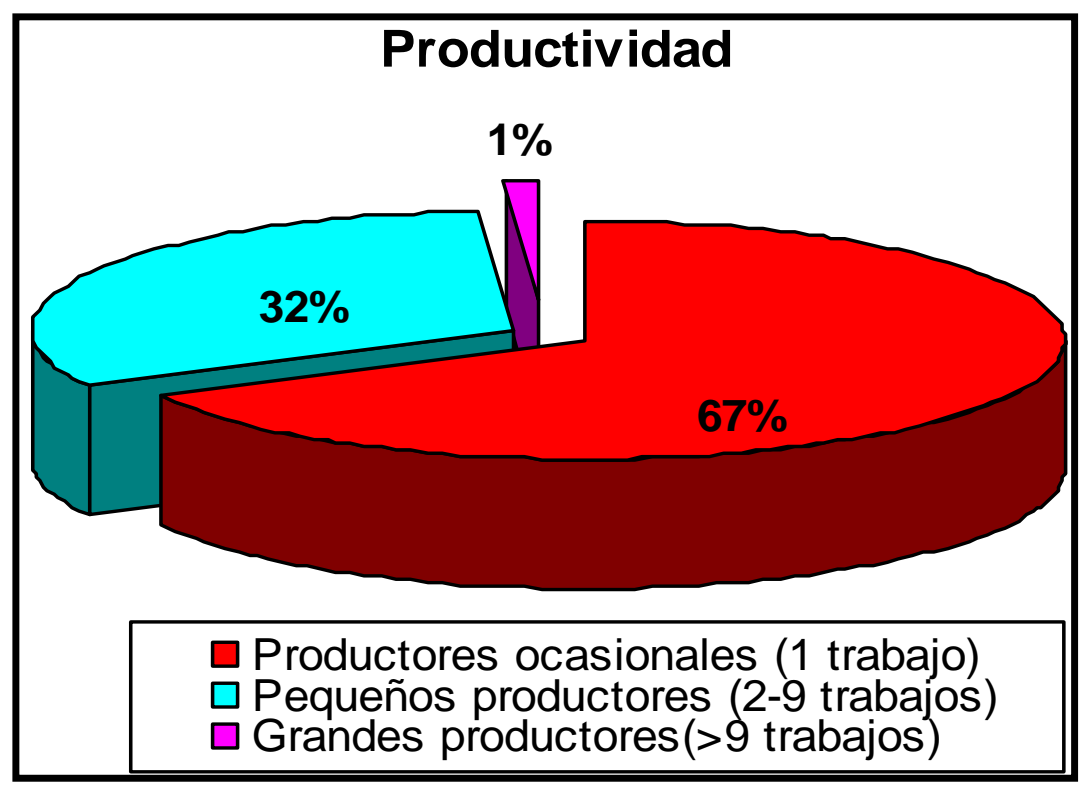

Gráfico 3: Distribución de autores según su productividad 
El $84 \%$ de los documentos se realizan sin colaboración institucional; 11 artículos se editan en colaboración con otras instituciones, entre las que destaca la Universidad de Huelva con 7 artículos. (Gráfico 4)

Gráfico 4: Distribución de las publicaciones según la colaboración institucional.

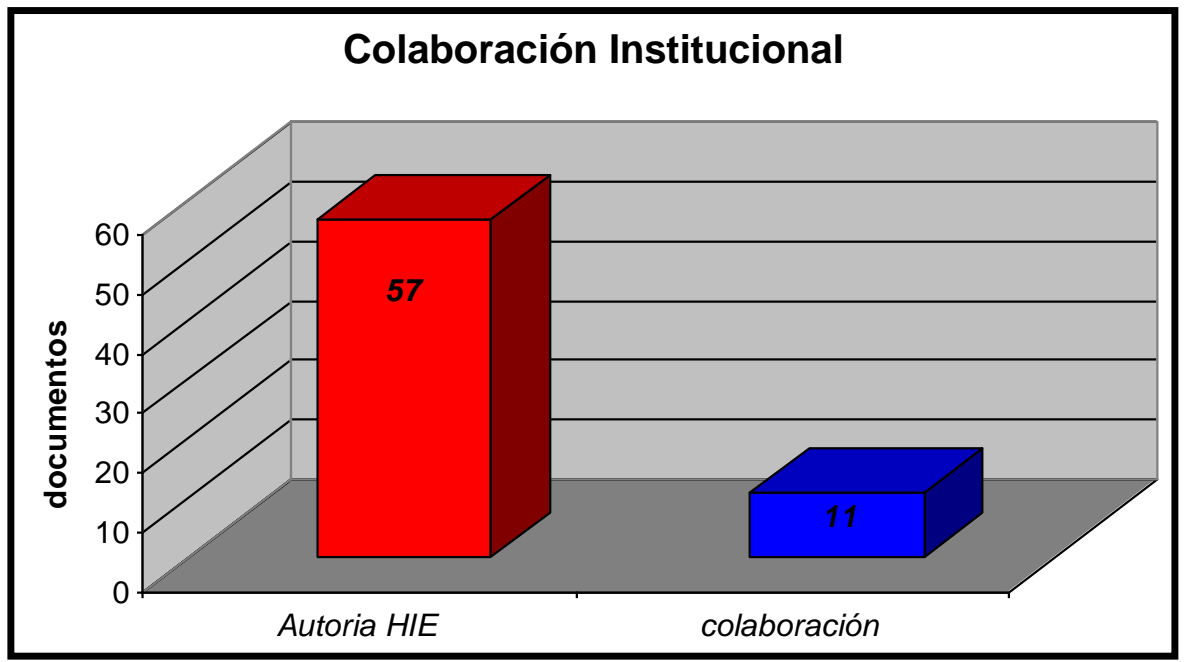

Se utilizan un promedio de 6,5 palabras clave por documento. Para los 68 trabajos se utilizan un total de 441 palabras clave, acumuladas en 240 descriptores diferentes. Las palabras clave más utilizadas son: Enfermería, Cuidados, Atención de enfermería, Cuidados de enfermería, Salud, Cirugía y Hospitales; suman el $20 \%$ del total; aunque existe una gran dispersión. (Gráfico 5). Todos los trabajos están en español.

Gráfico 5: Nube de las palabras clave utilizadas

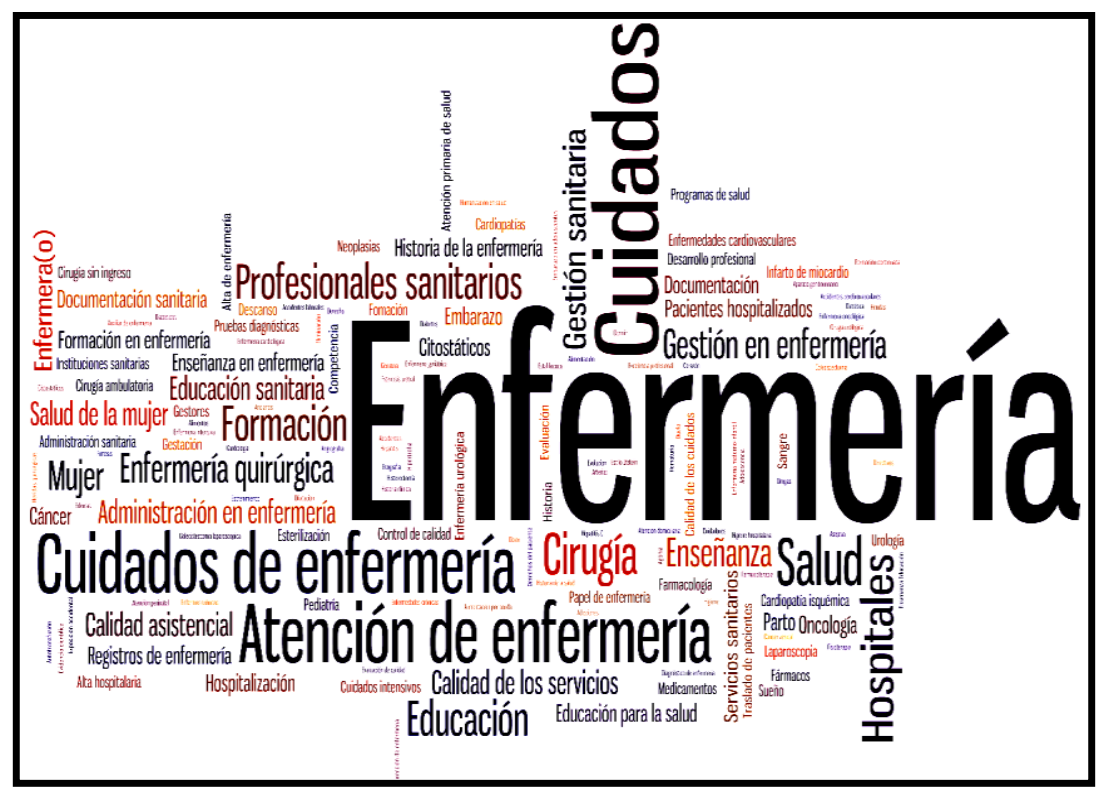




\section{CONCLUSIÓN-DISCUSIÓN}

A pesar de la distribución irregular anual de la producción científica de las Enfermeras del Hospital Infanta Elena de Huelva, se constata un crecimiento exponencial de las publicaciones (Gráfico 6). Se pone de manifiesto de este modo la Ley de Price ${ }^{19}$; ésta afirma que la literatura científica existente se duplica cada 10 o 15 años con un crecimiento exponencial.

Gráfico 6: Producción anual y acumulada del HIE

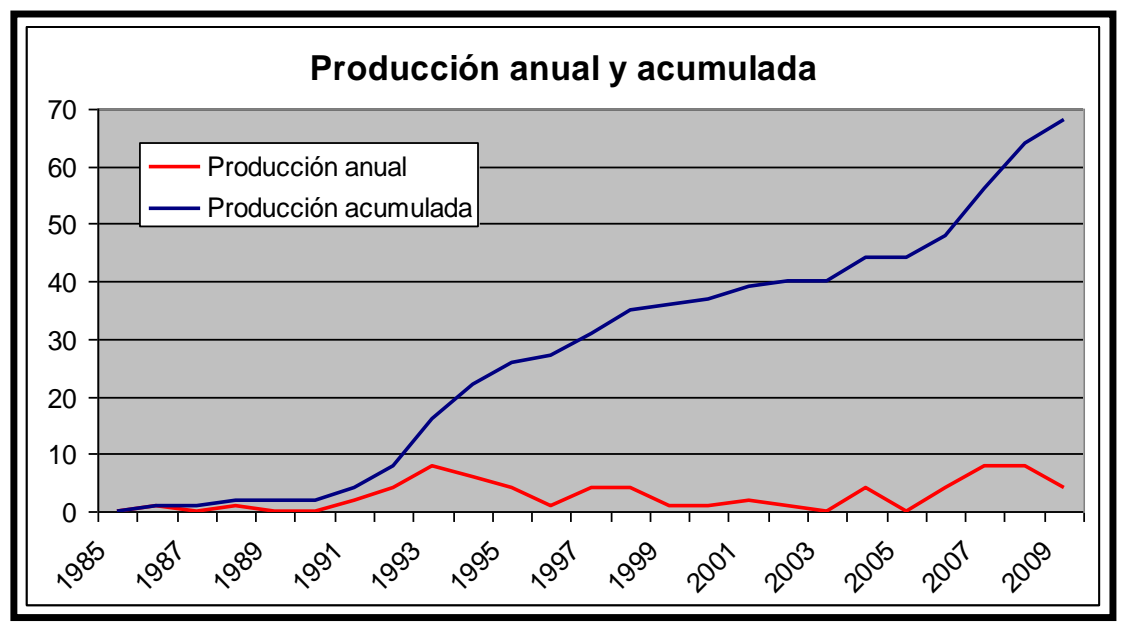

En los 25 años analizados encontramos un gran productor de literatura científica con más de 10 trabajos publicados entre el total de 98 autores que han publicado en este período. No se aprecia ningún grupo consolidado de investigación. Las áreas temáticas más publicadas son las relacionadas con la atención de enfermería y los cuidados.

Se observa una colaboración escasa tanto a nivel de autores firmantes como a nivel de instituciones. 11 documentos se elaboran en colaboración con otras instituciones. Son 2,4 autores los que firman cada documento. Analizando el número de firmas por artículo, se puede tener una idea del nivel de profesionalización de la comunidad científica y de la madurez de los autores. En la literatura científica hay una tendencia al aumento del número de autores y colaboradores en áreas experimentales y otras áreas que se encuentran en constante crecimiento, ya que se realizan investigaciones cada vez más complejas, especializadas y $\operatorname{costosas}^{20}$.

La falta de normalización de los nombres de los investigadores disminuye la visibilidad de los autores en las bases de datos bibliográficas.

El perfil del documento tipo publicado por las Enfermeras del HIE en los últimos 25 años es el siguiente:

- Autor que publica principalmente en los últimos 4 años.

- Publica 2 artículos en solitario en Documentos de Enfermería o Sesiones de Salud.

- Produce 1 artículo original y otro de revisión.

- Escribe en español con temática sobre Cuidados de Enfermería. 


\section{BIBLIOGRAFÍA}

1. Albornoz M. Estrategias para la promoción de las publicaciones científicas argentinas. Acta bioquím clín latinoam [revista en internet]. 2006 [citado 3 nov 2010]; 40(2):233-237. Disponible http://www.scielo.org.ar/scielo.php?script=sci_arttext\&pid=S0325$29572006000200012 \& \operatorname{lng}=\mathrm{es}$

2. Jiménez Contreras E. Las revistas científicas: el centro y la periferia. Rev Esp Doc Cient. 1992:15(2):174-182.

3. Aleixandre R, Giménez Sánchez JV, Terrada ML, López Piñero JM. Análisis de consumo de información en la revista Medicina Clínica. Med Clin (Barc). 1994;103:246-251.

4. Amezcua Martínez M, Gálvez Toro A, Cuesta de la Rosa R, Heierle Valero C, Poyatos Huertas E. La Pequeña Ciencia. Producción, Repercusión y Transferencia del Conocimiento. La Enfermería del área Lingüística del Español y del Portugués. Biblioteca Lascasas. Biblioteca Lascasas [revista en internet]. 2006 [citado 3 nov 2009];2(2). Disponible en: http://www.index-f.com/lascasas/documentos/lc0139.php

5. Rueda-Clausen Gómez CF, Villa-Roel Gutiérrez C, Rueda-Clausen Pinzón CE. Indicadores bibliométricos: origen, aplicación y nuevas propuestas. Med Unab. 2005;8(1):29-36.

6. Camps D, Samar ME, Ávila RE, Recuero Y. Estudio bibliométrico de un volumen de la revista Archivos de Medicina. Arch Med [revista en internet]. 2006 [Acceso 3 nov 2010];2(3). Disponible en: http: //archivosde medicina.com

7. Camps D, Recuero Y, Samar ME, Ávila R. Análisis bibliométrico de tesis de doctorado del área de las ciencias de la salud: Primera parte, Odontología. Rev Fac Cienc Med Univ Nac Córdoba. 2005;62:53-6.

8. Bordons M, Gómez Cl. La actividad científica española a través de indicadores bibliométricos en el período 1990-93. Rev Gen Inf Doc 1997; 7: 69-86.

9. Rousseau R. Indicadores bibliométricos y econométricos en la evaluación de instituciones científicas. ACIMED. 2001;9:23-9.

10. Camí J, Fernández MT, Gómez Caridad I. La producción científica española en biomedicina y salud. Un estudio a través del Science Citation Index (1986-1989). Med Clin (Barc). 1993;101:721-31.

11. Camí J, Zulueta MA, Fernández MT, Bordons M, Gómez I. Producción científica española en biomedicina y ciencias de la salud durante el periodo 1990-1993 (Science Citation Index y Social Science Citation Index) y comparación con el período 1986-1989. Med Clin (Barc). 1997;109:481-96.

12. Rodríguez Álvaro, M; Toledo Rosell, C. Análisis de la producción científica de la revista de enfermería ENE en los años 2007-2009. ENE, Revista de Enfermería. 2009;3(2):1224.

13. Balseiro Almario, Lasty; Torres Lagunas, Maria de los Ángeles; Zárate Grajales, Rosa.

Evaluación interna y externa de la Revista Enfermería Universitaria de la ENEO-UNAM durante el período 2004-2006. Enferm Universitaria -Mex-. 2009;6(1):12-21.

14. Jiménez Hernández JA, Ayuso García MD, Murillo Murillo R, Guillén Ríos JF. Evolución de las publicaciones periódicas españolas de enfermería. Análisis cuantitativo. Index de Enfermería. 2007;56.

15. Villar Álvarez F, Estrada Lorenzo JM, Pérez Andrés C, Rebollo Rodríguez MJ. Estudio bibliométrico de los artículos originales de la Revista Española de Salud Pública (19912000). Parte tercera: análisis de las referencias bibliográficas. Rev Esp Salud Publica [revista en la Internet]. 2007 [citado 3 nov 2010];81(3):247-259. Disponible en: http://www.scielosp.org/scielo.php?script=sci_arttext\&pid=S1135-

$57272007000300002 \& \operatorname{lng}=$ es.doi:10.590/\$1135-57272007000300002 
16. Muñoz Soler V, Flores López MJ, Cabañero Martínez MJ, Richart Martínez M. Análisis de referencias bibliográficas de originales publicados en revistas de enfermería nacionales e internacionales y de 2 disciplinas de la salud afines. Enferm Clínica. 2007;17(2):71-77.

17. Lozano-León T, Gómez-Fernández JA, Romero Indiano EM, Peral Belchior B. Producción científica de la Revista de la Sociedad Española de Enfermería Nefrológica durante los años 2005-2008. Rev Soc Esp Enferm Nefrol. 2010;13(4):223-227.

18. Sobrido Prieto M, Sobrido Prieto N, González Guitián C, Pichel Guerrero MJ, García Sánchez MM, Prieto Díaz A. Revistas españolas de Enfermería en bases de datos nacionales e internacionales. Index de Enfermería [revista en internet]. 2005 [citado 29 oct 2010];48-49. Disponible en: http://www.index-f.com/index-enfermeria/48-49revista/4849_articulo_74-77.php

19. De Solla Price DJ. Networks of scientic papers: The pattern of bibliographic references indicates the nature of the scientific research front. Science. 1965; 149(3683):510-515.

20. López Piñero JM, Terrada ML. Los indicadores bibliométricos y la evaluación de la actividad médico-científica (I). Usos y abusos de la bibliometría. Med Clin (Barc) 1992;98:64-8. 